\title{
Respuesta a la carta al editor Diagnóstico endoscópico de uncinariasis
}

\section{Response to a letter to the editor Endoscopic Diagnosis of Hookworms}

Gabriel Mosquera-Klinger, MD. ${ }^{1}$

\author{
Palabras clave \\ Uncinariasis, Trichuris tichiura, \\ antiparasitarios, endoscopia gas- \\ trointestinal.

\section{Keywords \\ Hookworm infections, Trichuris tichiura, antiparasitic agents, gas- trointestinal endoscopy.} \\ 1 Unidad de gastroenterología y endoscopia \\ digestiva. Hospital Pablo Tobón Uribe; Medellín, \\ Colombia. \\ *Correspondencia: Gabriel Mosquera-Klinger, MD \\ gami8203@yahoo.com \\ Fecha recibido: $17 / 04 / 20$ \\ Fecha aceptado: 25/04/20
}

De antemano, agradezco el interés demostrado por el caso clínico sobre el diagnóstico endoscópico de uncinariasis (1). Considero de gran relevancia el aporte y la profunda discusión académica basada en semiología endoscópica sobre el diagnóstico diferencial de las parasitosis intestinales planteada en la carta al editor.

Estoy de acuerdo en que la ubicación o el hábitat principal de las uncinarias es el tracto digestivo superior, en especial el intestino delgado (duodeno, yeyuno). Sin embargo, sí existe un número creciente de casos reportados en los cuales su localización se halla en la región colorrectal (2-4) e incluso en el apéndice cecal (5).

El Trichuris trichiura es el parásito intestinal que con más frecuencia se diagnostica en una colonoscopia y su hábitat usual es el colon, lo que constituye un diagnóstico diferencial que se debe considerar en este caso. Pero sí es fundamental destacar que el Trichuris trichiura recibe su nombre del griego Thrikhos, que significa pelo, dada la forma de la parte anterior del parásito, que es delgada y ocupa dos terceras partes de este.

Esto le brinda una característica típica de látigo: es muy delgado en su parte anterior y es allí donde está representada su mayor longitud. Así pues, la hembra termina en forma recta en su extremo posterior, mientras que el macho tiene una curvatura pronunciada y está provisto de una espícula copulatriz con un tamaño de entre 3 y $5 \mathrm{~cm}$ de longitud (6).

En cuanto a las características morfológicas de las uncinarias, se ha descrito que son parásitos cilíndricos, de color blanco, de entre 10-15 mm de longitud, con su extremo anterior generalmente recto y su cuerpo en curva amplia (con forma de $\mathrm{C}$ para el Ancylostoma duodenale y una ligera curva en sentido inverso a la parte anterior, que da apariencia de $\mathrm{S}$ para el Necator americanus). Estos hallazgos muy similares a los vistos en la Figura 1 del caso en discusión.

Con estas diferencias morfológicas descritas, concluimos que el T. trichiura macho tiene una curvatura pronunciada que le da una apariencia "enrollada", pero lo más característico de este parásito es su forma de látigo o pelo en su parte anterior (7) (Figura 2), que no se evidencia en nuestro caso.

Existen otros 2 datos clínicos que hacen poco probable la posibilidad de que los parásitos observados en la colonoscopia del caso en cuestión fueran T. trichiura. El primero es que el T. trichiura no es hematófago y los mecanismos que se acuñan a la anemia en esta parasitosis tienen relación con una lesión mecánica de la mucosa, dado que se introduce la porción anterior del parásito a la mucosa. Este traumatismo genera cambios inflamatorios locales y hemorragia, por lo cual los pacientes con frecuencia tienen síntomas como diarrea, sangrado, disentería e incluso presentan variaciones indistinguibles a una enfer- 


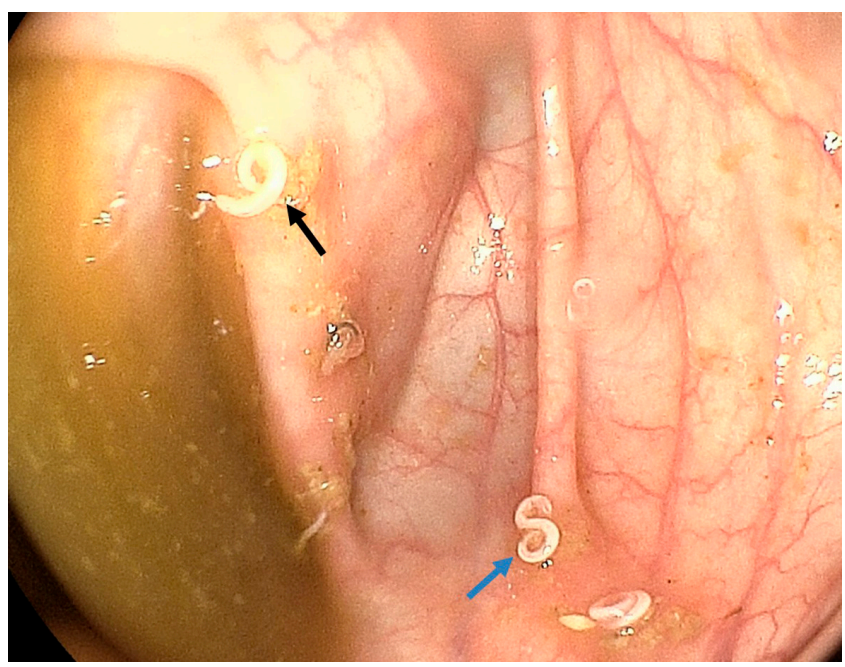

Figura 1. Múltiples uncinarias en el colon derecho. La flecha azul muestra la característica forma en $\mathrm{S}$ del $\mathrm{N}$. americanus, mientras que la flecha negra señala la característica forma en $\mathrm{C} \operatorname{del} A$. duodenale.

medad inflamatoria intestinal $(8,9)$, que no se presentaron en nuestro caso. La severidad de los cambios inflamatorios tiene una clara relación con la cantidad del parásito.

El otro aspecto que no está a favor de dicha hipótesis es la respuesta clínica tan buena, generada a partir del tratamiento con el pamoato de pirantel, tal y como se evidenció en nuestro paciente (1). El pamoato de pirantel y los benzimidazoles son los medicamentos de elección en la uncinariasis. Por tanto, se recomiendan esquemas de 3 días, cada 6 meses, durante 3 años. Así, el pamoato de pirantel tiene altas tasas de curación y de reducción de huevos para la uncinariasis. En contraste, no debe usarse para el T. trichiura, debido a su baja efectividad $(1,6)$.

Dentro de este contexto, para el caso del T. trichiura, se han recomendado históricamente los benzimidazoles, que son el tratamiento de elección, pese a que se ha descrito que con la terapia única se producen curaciones en menos del $75 \%$ (6). Las tasas de erradicación de huevos se podrían mejorar con esquemas combinados de benzimidazoles e ivermectina, según lo planteado en el trabajo de Knopp y colaboradores (10).

En este estudio, se describen unas tasas de erradicación significativas que alcanzan un $97 \%$ con la combinación entre mebendazol e ivermectina, y del $91 \%$ para albendazol e ivermectina (10). Sin embargo, es necesario precisar que

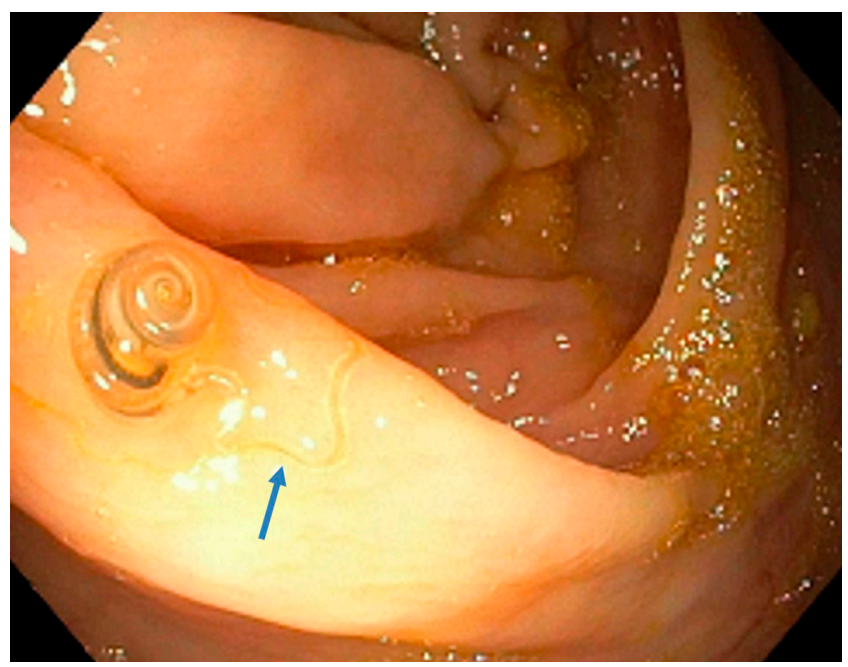

Figura 2. La flecha azul muestra la característica del parásito en forma de hilo o látigo en la parte anterior. Tomada de la referencia 7.

la ivermectina, como esquema único para estas parasitosis, tiene tasas de curación del $11 \%$ y de reducción de huevos del $59 \%(6,11)$. Por tanto, no se recomienda su uso para el T. trichiura, ni para las uncinarias en monoterapia.

Estamos de acuerdo con que en Colombia, en individuos provenientes de áreas rurales, se pueden presentar con frecuencia poliparasitosis y consideramos pertinente que, cuando existan dudas sobre el tipo de parásitos encontrados en estudios endoscópicos, se realice la extracción del parásito, a fin de aumentar las posibilidades de establecer el diagnóstico preciso.

Cuando se mide en años de vida ajustados en función de la discapacidad, la carga de morbilidad mundial por la uncinariasis supera a todas las demás enfermedades infecciosas tropicales importantes, con excepción del paludismo, la leishmaniasis y la filariasis linfática (4).

Por esta razón, me parece fundamental aclarar que las uncinarias son los helmintos que más se asocian a una anemia crónica grave, por lo que en todos los casos se debe tratar con antiparasitarios efectivos por al menos 3 años, tal y como se estableció en este paciente. En cambio, se ha descrito que en infecciones leves sin manifestaciones clínicas por T. trichiura no se requiere estrictamente un tratamiento (6). Las infecciones severas se deben tratar siempre. 
1. Pizza-Restrepo J, Mosquera-Klinger G. Diagnóstico endoscópico de uncinaria: presentación de un caso con anemia ferropénica grave. Rev Colomb Gastroenterol. 2019;34(4):433-437. http://doi.org/10.22516/25007440.289

2. Thomas V, Harish K, Tony J, Sunilkumar R, Ramachandran TM, Anitha PM. Colitis due to Ancylostoma duodenale. Indian J Gastroenterol. 2006;25(4):210-211.

3. Wang CH, Lee SC, Huang SS, Chang LC. Hookworm infection in a healthy adult that manifested as severe eosinphilia and diarrhea. J Microbiol Immunol Infect. 2011;44(6):484-487. http://doi.org/10.1016/j.jmii.2011.04.010

4. Hotez PJ, Bethony J, Bottazzi ME, Brooker S, Buss P. Hookworm: "the great infection of mankind". PLoS Med. 2005;2(3):e67. http://doi.org/10.1371/journal.pmed.0020067

5. Changela K, Sharma S, Reddy M. Ancylostoma duodenale: Lost Its way into Appendix. Am J Gastroenterol. 2017;112(suppl 1):S776-S778. https://doi.org/10.14309/00000434-201710001-01429

6. Botero D, Restrepo M. Parasitosis intestinales por nematodos. En: Botero D, Restrepo M. Parasitosis humanas. Medellín: Corporación para Investigaciones Biológicas; 1999. p. 139-60.

7. Sunkara T, Sharma SR, Ofosu A. Trichuris trichiura-An Unwelcome Surprise during Colonoscopy. Am J Trop Med
Hyg. 2018;99(3):555-556.

http://doi.org/10.4269/ajtmh.18-0209

8. Ok KS, Kim YS, Song JH, Lee JH, Ryu SH, Lee JH, Moon JS, Whang DH, Lee HK. Trichuris trichiura infection diagnosed by colonoscopy: case reports and review of literature. Korean J Parasitol. 2009;47(3):275-280. http://doi.org/10.3347/kjp.2009.47.3.275

9. Jha AK, Goenka MK, Suchismita A. Clinical correlates of trichuriasis diagnosed at colonoscopy. Indian J Gastroenterol. 2017;36(5):420-423. http://doi.org/10.1007/s12664-017-0795-8

10. Knopp S, Mohammed KA, Speich B, Hattendorf J, Khamis IS, Khamis AN, Stothard JR, Rollinson D, Marti H, Utzinger J. Albendazole and mebendazole administered alone or in combination with ivermectin against Trichuris trichiura: a randomized controlled trial. Clin Infect Dis. 2010;51(12):1420-1428. http://doi.org/10.1086/657310

11. Marti H, Haji HJ, Savioli L, Chwaya HM, Mgeni AF, Ameir JS, Hatz C. A comparative trial of a single-dose ivermectin versus three days of albendazole for treatment of Strongyloides stercoralis and other soil-transmitted helminth infections in children. Am J Trop Med Hyg. 1996;55(5):477-481. http://doi.org/10.4269/ajtmh.1996.55.477 